\title{
UNSTEADY STAGNATION POINT FLOW OF A NON-NEWTONIAN SECOND-GRADE FLUID
}

\author{
F. LABROPULU, X. XU, and M. CHINICHIAN
}

Received 5 December 2002

\begin{abstract}
The unsteady two-dimensional flow of a viscoelastic second-grade fluid impinging on an infinite plate is considered. The plate is making harmonic oscillations in its own plane. A finite difference technique is employed and solutions for small and large frequencies of the oscillations are obtained.
\end{abstract}

2000 Mathematics Subject Classification: 65L06, 65L12, 76D05.

1. Introduction. In the past two decades, the importance of non-Newtonian viscoelastic liquids have become evident due to their occurrence in industrial processes. Behaviour of viscoelastic fluids cannot be accurately described by the Newtonian fluid model. The equations of motion of viscoelastic fluids are highly nonlinear and one order higher than the Navier-Stokes equations.

The two-dimensional stagnation point flow is an interesting problem in the history of fluid dynamics and has received considerable attention. Beard and Walters [2] used boundary-layer equations to study two-dimensional flow near a stagnation point of a viscoelastic fluid. Dorrepaal et al. [3] investigated the behavior of a viscoelastic fluid impinging on a flat rigid wall at an arbitrary angle of incidence. Labropulu et al. [5] studied the oblique flow of a viscoelastic fluid impinging on a porous wall with suction or blowing.

Unsteady stagnation point flow of a Newtonian fluid has also been studied extensively. Rott [8] and Glauert [4] have studied the stagnation point flow of a Newtonian fluid when the plate performs harmonic oscillations in its own plane. Srivastava [9] has studied the same problem for a non-Newtonian second-grade fluid. He used the Karman-Pohlhausen method to solve the resulting equations.

This paper considers the unsteady two-dimensional flow of an incompressible viscoelastic second-grade fluid impinging on an infinite flat plate. We assume that the plate is making harmonic oscillations in its own plane. Series method is employed to evaluate the solution for small and large frequencies of the oscillations. The resulting differential equations are solved numerically using a finite difference method developed by Ariel [1].

2. Flow equations. The flow of a viscous incompressible non-Newtonian second-grade fluid, neglecting thermal effects and body forces, is governed 
by

$$
\operatorname{div} \underset{\sim}{V}=0, \quad \rho \underset{\sim}{\dot{V}}=\operatorname{div} \underset{\approx}{T}
$$

when the constitutive equation for the Cauchy stress tensor $\underset{\approx}{T}$ which describes second-grade fluids given by Rivlin and Ericksen [7] is

$$
\begin{gathered}
\underset{\approx}{T}=-p \underset{\approx}{I}+\mu \underset{\approx}{A_{1}}+\alpha_{1} \underset{\approx}{A_{2}}+\alpha_{2} \underset{\approx}{A_{1}^{2}}, \quad \underset{\approx}{A_{1}}=(\operatorname{grad} \underset{\sim}{V})+(\operatorname{grad} V)^{T}, \\
\underset{\approx}{A_{2}}=\dot{\approx}_{1}+(\operatorname{grad} V)^{T} \underset{\approx}{A_{1}}+\underset{\approx}{A_{1}}(\operatorname{grad} V) .
\end{gathered}
$$

Here $V$ is the velocity vector field, $p$ the fluid pressure function, $\rho$ the constant fluid density, $\mu$ the constant coefficient of viscosity, and $\alpha_{1}, \alpha_{2}$ the normal stress moduli.

Considering the flow to be plane, we take $V=(u(x, y, t), v(x, y, t))$ and $p=p(x, y, t)$ so that our flow equations (2.1) and (2.2) take the form

$$
\begin{aligned}
\frac{\partial u}{\partial x}+ & \frac{\partial v}{\partial y}=0, \\
\frac{\partial u}{\partial t}+ & u \frac{\partial u}{\partial x}+v \frac{\partial u}{\partial y}+\frac{1}{\rho} \frac{\partial p}{\partial x} \\
= & v \nabla^{2} u+\frac{\alpha_{1}}{\rho}\left\{\frac{\partial}{\partial t}\left(\nabla^{2} u\right)\right. \\
& +\frac{\partial}{\partial x}\left[2 u \frac{\partial^{2} u}{\partial x^{2}}+2 v \frac{\partial^{2} u}{\partial x \partial y}+4\left(\frac{\partial u}{\partial x}\right)^{2}+2 \frac{\partial v}{\partial x}\left(\frac{\partial v}{\partial x}+\frac{\partial u}{\partial y}\right)\right] \\
& \left.+\frac{\partial}{\partial y}\left[\left(u \frac{\partial}{\partial x}+v \frac{\partial}{\partial y}\right)\left(\frac{\partial v}{\partial x}+\frac{\partial u}{\partial y}\right)+2 \frac{\partial u}{\partial x} \frac{\partial u}{\partial y}+2 \frac{\partial v}{\partial x} \frac{\partial v}{\partial y}\right]\right\} \\
+ & \frac{\alpha_{2}}{\rho} \frac{\partial}{\partial x}\left[4\left(\frac{\partial u}{\partial x}\right)^{2}+\left(\frac{\partial v}{\partial x}+\frac{\partial u}{\partial y}\right)^{2}\right],
\end{aligned}
$$

$$
\begin{aligned}
& \frac{\partial v}{\partial t}+ u \frac{\partial v}{\partial x}+v \frac{\partial v}{\partial y}+ \\
&=v \nabla^{2} v+\frac{1}{\rho} \frac{\alpha_{1}}{\rho}\left\{\frac{\partial}{\partial t}\left(\nabla^{2} v\right)\right. \\
&+\frac{\partial}{\partial x}\left[\left(u \frac{\partial}{\partial x}+v \frac{\partial}{\partial y}\right)\left(\frac{\partial v}{\partial x}+\frac{\partial u}{\partial y}\right)+2 \frac{\partial u}{\partial x} \frac{\partial u}{\partial y}+2 \frac{\partial v}{\partial x} \frac{\partial v}{\partial y}\right] \\
&\left.+\frac{\partial}{\partial y}\left[2 u \frac{\partial^{2} v}{\partial x \partial y}+2 v \frac{\partial^{2} v}{\partial y^{2}}+4\left(\frac{\partial v}{\partial y}\right)^{2}+2 \frac{\partial u}{\partial y}\left(\frac{\partial v}{\partial x}+\frac{\partial u}{\partial y}\right)\right]\right\} \\
&+ \frac{\alpha_{2}}{\rho} \frac{\partial}{\partial y}\left[4\left(\frac{\partial v}{\partial y}\right)^{2}+\left(\frac{\partial v}{\partial x}+\frac{\partial u}{\partial y}\right)^{2}\right]
\end{aligned}
$$

where $v=\mu / \rho$ is the kinematic viscosity. 
The continuity equation (2.3) implies the existence of a stream function $\psi(x, y, t)$ such that

$$
u=\frac{\partial \psi}{\partial y}, \quad v=-\frac{\partial \psi}{\partial x}
$$

Substitution of (2.6) in (2.4) and (2.5) and elimination of pressure from the resulting equations using $p_{x y}=p_{y x}$ yields

$$
\frac{\partial}{\partial t}\left(\nabla^{2} \psi\right)-\frac{\alpha_{1}}{\rho} \frac{\partial}{\partial t}\left(\nabla^{4} \psi\right)-\frac{\partial\left(\psi, \nabla^{2} \psi\right)}{\partial(x, y)}+\frac{\alpha_{1}}{\rho} \frac{\partial\left(\psi, \nabla^{4} \psi\right)}{\partial(x, y)}-v \nabla^{4} \psi=0 .
$$

Having obtained a solution of (2.7), the velocity components are given by (2.6) and the pressure can be found by integrating (2.4) and (2.5).

The shear stress component $\tau_{12}$ of the Cauchy stress $\underset{\approx}{T}$ is given by

$$
\begin{aligned}
\tau_{12}=\mu\left\{\frac{\partial^{2} \psi}{\partial y^{2}}-\frac{\partial^{2} \psi}{\partial x^{2}}+\alpha_{1}[\right. & \frac{\partial \psi}{\partial y}\left(\frac{\partial^{3} \psi}{\partial x \partial y^{3}}-\frac{\partial^{3} \psi}{\partial x^{3}}\right)-\frac{\partial \psi}{\partial x}\left(\frac{\partial^{3} \psi}{\partial y^{3}}-\frac{\partial^{3} \psi}{\partial x^{2} \partial y}\right) \\
& \left.\left.+2 \frac{\partial^{2} \psi}{\partial x \partial y} \frac{\partial^{2} \psi}{\partial y^{2}}+2 \frac{\partial^{2} \psi}{\partial x^{2}} \frac{\partial^{2} \psi}{\partial x \partial y}\right]\right\} .
\end{aligned}
$$

3. Solutions. We consider the two-dimensional flow of an incompressible fluid against an infinite plate normal to the flow. We assume that the plate makes harmonic oscillations on its own plane and its velocity in the $x$-direction is $a e^{i \omega t}$ where $a$ and $\omega$ are constants.

The boundary conditions are then given by

$$
\begin{gathered}
\frac{\partial \psi}{\partial y}=a e^{i \omega t}, \quad \frac{\partial \psi}{\partial x}=0 \quad \text { at } y=0, \\
\frac{\partial \psi}{\partial y}=c x \quad \text { as } y \longrightarrow \infty .
\end{gathered}
$$

Following Glauert [4], we assume that

$$
\psi=c x f(y)+a e^{i \omega t} g(y) .
$$

The boundary conditions take the form

$$
\begin{gathered}
f(0)=f^{\prime}(0)=0, \quad g^{\prime}(0)=1, \\
f^{\prime}(\infty)=1, \quad g^{\prime}(\infty)=0 .
\end{gathered}
$$

Using (3.2) in (2.7), we obtain

$$
\begin{gathered}
v f^{(i v)}+c\left(f f^{\prime \prime \prime}-f^{\prime} f^{\prime \prime}\right)-\frac{\alpha_{1} c}{\rho}\left(f f^{(v)}-f^{\prime} f^{(i v)}\right)=0 \\
v g^{(i v)}-i \omega g^{\prime \prime}+\frac{\alpha_{1}}{\rho} i \omega g^{(i v)}+c\left(f g^{\prime \prime \prime}-f^{\prime \prime} g^{\prime}\right)-\frac{\alpha_{1} c}{\rho}\left(f g^{(v)}-f^{(i v)} g^{\prime}\right)=0
\end{gathered}
$$


TABLE 3.1. Numerical values of $F^{\prime \prime}(0), \phi_{0}^{\prime}(0), \phi_{1}^{\prime}(0)$, and $\phi_{2}^{\prime}(0)$ for different values of $W_{e}$.

\begin{tabular}{llllr}
\hline$W_{e}$ & \multicolumn{1}{c}{$F^{\prime \prime}(0)$} & \multicolumn{1}{c}{$\phi_{0}^{\prime}(0)$} & $\phi_{1}^{\prime}(0)$ & \multicolumn{1}{c}{$\phi_{2}^{\prime}(0)$} \\
\hline 0.0 & 1.23259 & -0.811318 & -0.49307 & 0.0945488 \\
0.1 & 1.36954 & -0.86709 & -0.547302 & 0.0658565 \\
0.2 & 1.5873 & -0.947485 & -0.633897 & 0.0221985 \\
0.3 & 2.11092 & -1.10879 & -0.842867 & -0.0761073 \\
\hline
\end{tabular}

Nondimensionalizing using

$$
\eta=\sqrt{\frac{c}{v}} y, \quad f(y)=\sqrt{\frac{\nu}{c}} F(\eta), \quad g(y)=\sqrt{\frac{\nu}{c}} G(\eta)
$$

we get

$$
\begin{gathered}
F^{(i v)}+F F^{\prime \prime \prime}-F^{\prime} F^{\prime \prime}+W_{e}\left(F F^{(v)}-F^{\prime} F^{(i v)}\right)=0 \\
G^{(i v)}+F G^{\prime \prime \prime}-F^{\prime \prime} G^{\prime}+W_{e}\left(F G^{(v)}-F^{(i v)} G^{\prime}\right)-\frac{i \omega}{c} G^{\prime \prime}-\frac{i \omega W_{e}}{c} G^{(i v)}=0
\end{gathered}
$$

where $W_{e}=-\alpha_{1} c / \rho v$ is the Weissenberg number.

Integrating (3.6) once with respect to $\eta$ and using the conditions at infinity, we have

$$
\begin{gathered}
F^{\prime \prime \prime}+F F^{\prime \prime}-F^{2}+W_{e}\left(F F^{(i v)}-2 F^{\prime} F^{\prime \prime \prime}+F^{\prime 2}\right)=-1, \\
F(0)=0, \quad F^{\prime}(0)=0, \quad F^{\prime}(\infty)=1, \\
G^{\prime \prime \prime}+F G^{\prime \prime}-F^{\prime} G^{\prime}+W_{e}\left(F G^{(i v)}-F^{\prime} G^{\prime \prime \prime}+F^{\prime \prime} G^{\prime \prime}-F^{\prime \prime \prime} G^{\prime}\right)-\frac{i \omega}{c}\left(G^{\prime}+W_{e} G^{\prime \prime \prime}\right)=0, \\
G^{\prime}(0)=1, \quad G^{\prime}(\infty)=0 .
\end{gathered}
$$

System (3.7) has been solved numerically by many authors (Beard and Walters [2] and Ariel [1]). Using the shooting method with the finite difference technique described by Ariel [1], we find that $F^{\prime \prime}(0)=1.23259$ when $W_{e}=0$. Numerical values of $F^{\prime \prime}(0)$ for different values of $W_{e}$ are shown in Table 3.1. Figure 3.1 shows the profiles of $F^{\prime}$ for various $W_{e}$. We observed that as the elasticity of the fluid increases, the velocity near the wall increases. Figure 3.2 depicts the profiles of $F$ for various $W_{e}$.

Letting $\phi(\eta)=G^{\prime}(\eta)$, then system (3.8) becomes

$$
\begin{gathered}
\phi^{\prime \prime}+F \phi^{\prime}-F^{\prime} \phi+W_{e}\left(F \phi^{\prime \prime \prime}-F^{\prime} \phi^{\prime \prime}+F^{\prime \prime} \phi^{\prime}-F^{\prime \prime \prime} \phi\right)-\frac{i \omega}{c}\left(\phi+W_{e} \phi^{\prime \prime}\right)=0 \\
\phi(0)=1, \quad \phi(\infty)=0 .
\end{gathered}
$$




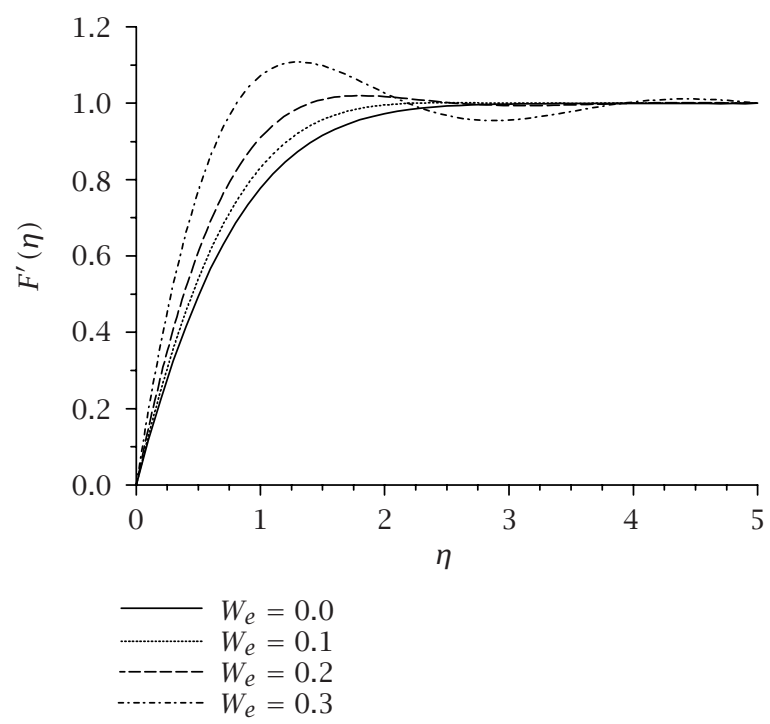

FIGURE 3.1. Variation of $F^{\prime}(\eta)$ with $W_{e}$.

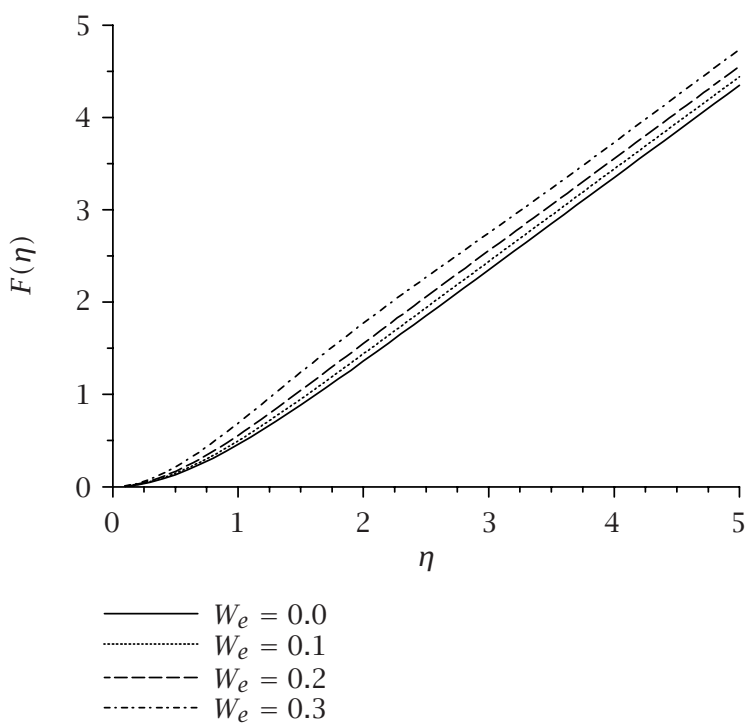

FIGURE 3.2. Variation of $F(\eta)$ with $W_{e}$.

The only parameter in (3.9) is the frequency ratio $\omega / c$. Series solutions will be developed, valid for small and large values of $\omega / c$, respectively. 


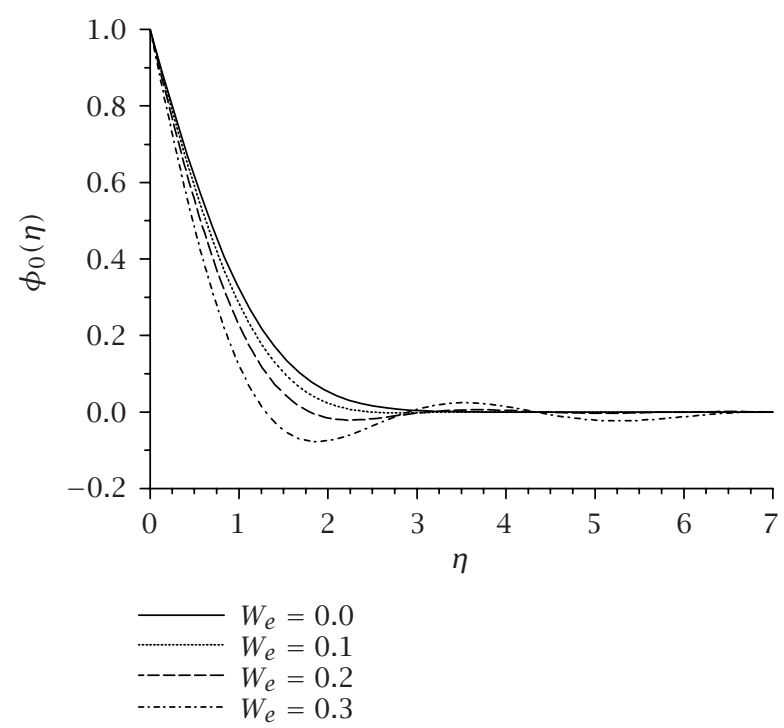

FIGURE 3.3. Variation of $\phi_{0}(\eta)$ with $W_{e}$.

3.1. Small values of $\omega / c$. Consider the case where $\omega=0$, which implies that the plate velocity has the constant value $a$. Letting $\phi=\phi_{0}$, then system (3.9) gives

$$
\begin{gathered}
\phi_{0}^{\prime \prime}+F \phi_{0}^{\prime}-F^{\prime} \phi_{0}+W_{e}\left(F \phi_{0}^{\prime \prime \prime}-F^{\prime} \phi_{0}^{\prime \prime}+F^{\prime \prime} \phi_{0}^{\prime}-F^{\prime \prime \prime} \phi_{0}\right)=0 \\
\phi_{0}(0)=1, \quad \phi_{0}(\infty)=0
\end{gathered}
$$

This system is solved numerically by using a shooting method and it is found that for $W_{e}=0, \phi_{0}^{\prime}(0)=-0.811318$ which is in good agreement with the value obtained by Glauert [4]. Numerical values of $\phi_{0}^{\prime}(0)$ for different values of $W_{e}$ are shown in Table 3.1. Figure 3.3 depicts the profiles of $\phi_{0}$ for various values of $W_{e}$.

For small but nonzero values of $\omega / c$, we let

$$
\phi(\eta)=\sum_{n=0}^{\infty}\left(\frac{i \omega}{c}\right)^{n} \phi_{n}(\eta)=\phi_{0}(\eta)+\frac{i \omega}{c} \phi_{1}(\eta)+\left(\frac{i \omega}{c}\right)^{2} \phi_{2}(\eta)+\cdots
$$

Substituting (3.11) into (3.9), we get, for $n \geq 1$,

$$
\begin{gathered}
\phi_{n}^{\prime \prime}+F \phi_{n}^{\prime}-F^{\prime} \phi_{n}+W_{e}\left(F \phi_{n}^{\prime \prime \prime}-F^{\prime} \phi_{n}^{\prime \prime}+F^{\prime \prime} \phi_{n}^{\prime}-F^{\prime \prime \prime} \phi_{n}\right)=\phi_{n-1}+W_{e} \phi_{n-1}^{\prime \prime}, \\
\phi_{n}(0)=0, \quad \phi_{n}(\infty)=0 .
\end{gathered}
$$




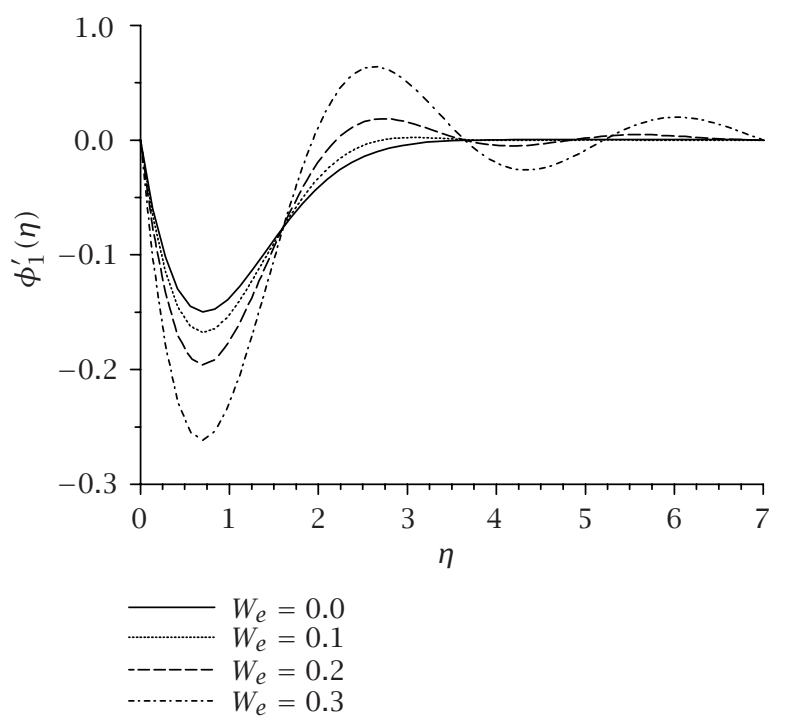

FIGURE 3.4. Variation of $\phi_{1}^{\prime}(\eta)$ with $W_{e}$.

This system can be solved numerically either by using the perturbation technique or by a finite difference scheme. Numerical integration of system (3.12) for $n=1$ using a finite difference technique gives, for $W_{e}=0, \phi_{1}^{\prime}(0)=$ -0.49307 which is in good agreement with Glauert's value [4]. Numerical values of $\phi_{1}^{\prime}(0)$ for different values of $W_{e}$ are shown in Table 3.1. Figure 3.4 shows the profiles of $\phi_{1}$ for various values of $W_{e}$.

Numerical integration of system (3.12) for $n=2$ using a finite difference technique gives, for $W_{e}=0, \phi_{2}^{\prime}(0)=0.0945488$ which is in good agreement with Glauert's value [4]. Numerical values of $\phi_{2}^{\prime}(0)$ for different values of $W_{e}$ are shown in Table 3.1. Figure 3.5 depicts the profiles of $\phi_{2}$ for various values of $W_{e}$.

The oscillating component of the shear stress on the wall is given by

$$
\frac{\tau_{12}}{\rho a^{2}}=\sqrt{\frac{c \mathcal{V}}{a^{2}}} e^{i \omega t}\left[\phi_{0}^{\prime}(0)+\frac{i \omega}{c} \phi_{1}^{\prime}(0)-W_{e} F^{\prime \prime}(0)\right],
$$

where $F^{\prime \prime}(0), \phi_{0}^{\prime}(0)$, and $\phi_{1}^{\prime}(0)$ are given in Table 3.1 for different values of $W_{e}$. When $W_{e}=0$, the value of the shear stress on the wall is in good agreement with the value obtained by Glauert [4].

3.2. Large values of $\omega / c$. When $\omega / c$ is large, we let

$$
Y=\sqrt{\frac{i \omega}{c}} \eta=\sqrt{\frac{i \omega}{v}} y .
$$




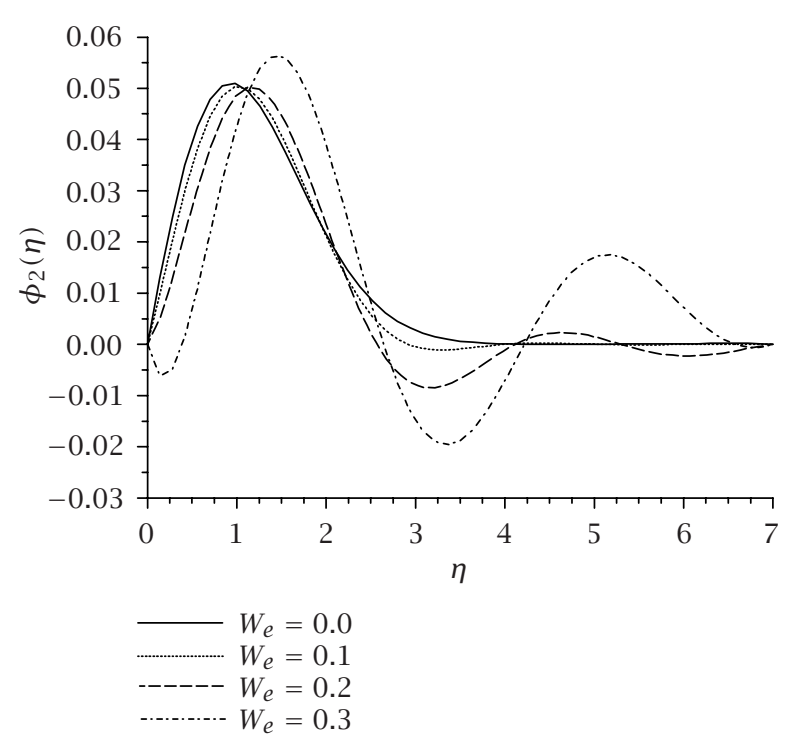

FIGURE 3.5. Variation of $\phi_{2}(\eta)$ with $W_{e}$.

Letting $\sqrt{i \omega / c}=\alpha$, then $d / d \eta=d / \alpha d Y$ and (3.9) takes the form

$$
\begin{aligned}
& \frac{1}{\alpha^{2}} \frac{d^{2} \phi}{d Y^{2}}+\frac{1}{\alpha}\left[F \frac{d \phi}{d Y}-\frac{d F}{d Y} \phi\right] \\
& \quad+\frac{1}{\alpha^{3}} W_{e}\left[F \frac{d^{3} \phi}{d Y^{3}}-\frac{d F}{d Y} \frac{d^{2} \phi}{d Y^{2}}+\frac{d^{2} F}{d Y^{2}} \frac{d \phi}{d Y}-\frac{d^{3} F}{d Y^{3}} \phi\right]-\frac{1}{\alpha^{2}} \phi-\frac{W_{e}}{\alpha^{4}} \frac{d^{2} \phi}{d Y^{2}}=0 .
\end{aligned}
$$

Since $W_{e}$ is small for most fluids which behave as second-order fluids (see Markovitz and Coleman [6]), we follow Srivastava [9] and take $W_{e}$ to be of the order of $\alpha^{2}$. Thus, $W_{e}=m \alpha^{2}$ and (3.15) becomes

$$
\begin{aligned}
(1-m) & \frac{d^{2} \phi}{d Y^{2}}+\alpha\left[F \frac{d \phi}{d Y}-\frac{d F}{d Y} \phi\right] \\
& +m \alpha\left[F \frac{d^{3} \phi}{d Y^{3}}-\frac{d F}{d Y} \frac{d^{2} \phi}{d Y^{2}}+\frac{d^{2} F}{d Y^{2}} \frac{d \phi}{d Y}-\frac{d^{3} F}{d Y^{3}} \phi\right]-\phi=0 .
\end{aligned}
$$

The expansion for $F(\eta)$ near the wall $\eta=0$ is

$$
F(\eta)=\frac{1}{2} A \eta^{2}+\frac{1}{6}\left(-1-W_{e} A^{2}\right) \eta^{3}+\frac{1}{120} A^{2} \eta^{5}+\frac{1}{720}\left(-2 A-W_{e} A^{3}\right) \eta^{6}+\cdots,
$$


where $A=F^{\prime \prime}(0)$. Since $\eta=\alpha Y$ and $W_{e}=m \alpha^{2}$, the above expansion takes the form

$$
\begin{aligned}
F(Y)= & \frac{1}{2} A \alpha^{2} Y^{2}+\frac{1}{6}\left(-1-m \alpha^{2} A^{2}\right) \alpha^{3} Y^{3} \\
& +\frac{1}{120} A^{2} \alpha^{5} Y^{5}-\frac{1}{720}\left(2 A+m \alpha^{2} A^{2}\right) \alpha^{6} Y^{6}+\cdots
\end{aligned}
$$

Since for large values of $\omega / c$ the parameter $\alpha$ is small, we let

$$
\phi=\sum_{n=0}^{\infty} \alpha^{n} \phi_{n}(Y)=\phi_{0}(Y)+\alpha \phi_{1}(Y)+\alpha^{2} \phi_{2}(Y)+\cdots .
$$

The boundary conditions are

$$
\phi_{0}(0)=1, \quad \phi_{n}(0)=0 \quad \text { if } n \geq 1, \quad \phi_{n}(\infty)=0 \quad \forall n .
$$

Substituting (3.19) in (3.16) and equating the coefficients of different powers of $\alpha$ to zero, we find that the boundary value problem for $\phi_{0}(Y)$ is

$$
(1-m) \frac{d^{2} \phi_{0}}{d Y^{2}}-\phi_{0}=0, \quad \phi_{0}(0)=1, \quad \phi_{0}(\infty)=0,
$$

with solution $\phi_{0}(Y)=\exp [-Y / \sqrt{1-m}]$ provided $m \neq 1$.

The second and third equations give that $\phi_{1}$ and $\phi_{2}$ are zero. The next four equations for $\phi_{3}(Y), \phi_{4}(Y), \phi_{5}(Y)$, and $\phi_{6}(Y)$ are

$$
\begin{aligned}
(1-m) \frac{d^{2} \phi_{3}}{d Y^{2}}-\phi_{3}= & -\frac{1}{2} m A Y^{2} \frac{d^{3} \phi_{0}}{d Y^{3}}+m A Y \frac{d^{2} \phi_{0}}{d Y^{2}} \\
& +\left(-\frac{1}{2} A Y^{2}-m A\right) \frac{d \phi_{0}}{d Y}+A Y \phi_{0}, \\
(1-m) \frac{d^{2} \phi_{4}}{d Y^{2}}-\phi_{4}= & \frac{1}{6} m Y^{3} \frac{d^{3} \phi_{0}}{d Y^{3}}+\left(\frac{1}{6} Y^{3}-m Y\right) \frac{d \phi_{0}}{d Y}+\left(-\frac{1}{2} Y^{2}-m\right) \phi_{0}, \\
(1-m) \frac{d^{2} \phi_{5}}{d Y^{2}}-\phi_{5}= & 0, \\
(1-m) \frac{d^{2} \phi_{6}}{d Y^{2}}-\phi_{6}= & \left(\frac{1}{24} A^{2} Y^{4}-m^{2} A^{2}\right) \phi_{0} \\
& +\left(\frac{1}{3} m A^{2} Y^{3}-\frac{1}{120} A^{2} Y^{5}-m^{2} A^{2} Y\right) \frac{d \phi_{0}}{d Y} \\
& +\left(-\frac{1}{2} m^{2} A^{2} Y^{2}+\frac{1}{24} m A^{2} Y^{4}\right) \frac{d^{2} \phi_{0}}{d Y^{2}} \\
& +\left(\frac{1}{6} m^{2} A^{2} Y^{3}-\frac{1}{120} m A^{2} Y^{5}\right) \frac{d^{3} \phi_{0}}{d Y^{3}} \\
& +A Y \phi_{3}+\left(-\frac{1}{2} A Y^{2}+m A\right) \frac{d \phi_{3}}{d Y} \\
& +m A Y \frac{d^{2} \phi_{3}}{d Y^{2}}-\frac{1}{2} m A Y^{2} \frac{d^{3} \phi_{3}}{d Y^{3}} .
\end{aligned}
$$


Solving these equations and using the boundary conditions, we obtain

$$
\begin{aligned}
& \phi_{3}(Y)=-\frac{A}{1-m} e^{-Y / \sqrt{1-m}}\left[\frac{3-4 m}{8} Y+\frac{3}{8 \sqrt{1-m}} Y^{2}+\frac{1}{12(1-m)} Y^{3}\right] \text {, } \\
& \phi_{4}(Y)=e^{-Y / \sqrt{1-m}}\left[\frac{3+4 m}{16 \sqrt{1-m}} Y+\frac{3-4 m}{16(1-m)} Y^{2}\right. \\
& \left.+\frac{1}{8(1-m) \sqrt{1-m}} Y^{3}+\frac{1}{48(1-m)^{2}} Y^{4}\right] \text {, } \\
& \phi_{5}(Y)=0, \\
& \phi_{6}(Y)=e^{-Y / \sqrt{1-m}}\left[-\frac{\left(40 m^{3}-50 m^{2}+28 m-33\right) A^{2}}{128(1-m) \sqrt{1-m}} Y\right. \\
& +\frac{\left(24 m^{3}+18 m^{2}-52 m+33\right) A^{2}}{128(1-m)^{2}} Y^{2} \\
& -\frac{\left(8 m^{3}-2 m^{2}+64 m-33\right) A^{2}}{196(1-m)^{2} \sqrt{1-m}} Y^{3} \\
& +\frac{\left(8 m^{3}-30 m^{2}-36 m+27\right) A^{2}}{384(1-m)^{3}} Y^{4} \\
& \left.-\frac{\left(3 m^{2}+6 m-9\right) A^{2}}{480(1-m)^{3} \sqrt{1-m}} Y^{5}-\frac{\left(m^{2}-2 m-4\right) A^{2}}{1440(1-m)^{4}} Y^{6}\right],
\end{aligned}
$$

provided $m \neq 1$. If $m=0$, we recover the solutions for the Newtonian fluid obtained by Glauert [4].

The oscillating component of the shear stress on the wall is given by

$$
\begin{aligned}
\frac{\tau_{12}}{\rho a^{2}}=-\sqrt{\frac{c \nu}{a^{2}}}[ & \frac{1}{\alpha \sqrt{1-m}}+\frac{(3-4 m) A}{8(1-m)} \alpha^{2}-\frac{3+4 m}{16 \sqrt{1-m}} \alpha^{3} \\
& \left.+\frac{\left(40 m^{3}-50 m^{2}+28 m-33\right) A^{2}}{128(1-m) \sqrt{1-m}} \alpha^{5}-W_{e} A\right] .
\end{aligned}
$$

If $m=0$, the shear stress is in good agreement with the result obtained by Glauert [4].

\section{REFERENCES}

[1] P. D. Ariel, A hybrid method for computing the flow of viscoelastic fluids, Internat. J. Numer. Methods Fluids 14 (1992), no. 7, 757-774.

[2] D. W. Beard and K. Walters, Elastico-viscous boundary-layer flows. I. Twodimensional flow near a stagnation point, Proc. Cambridge Philos. Soc. 60 (1964), 667-674.

[3] J. M. Dorrepaal, O. P. Chandna, and F. Labropulu, The flow of a visco-elastic fluid near a point of re-attachment, Z. Angew. Math. Phys. 43 (1992), no. 4, 708714.

[4] M. B. Glauert, The laminar boundary layer on oscillating plates and cylinders, J. Fluid Mech. 1 (1956), 97-110.

[5] F. Labropulu, J. M. Dorrepaal, and O. P. Chandna, Viscoelastic fluid flow impinging on a wall with suction or blowing, Mech. Res. Comm. 20 (1993), no. 2, 143153. 
[6] H. Markovitz and B. D. Coleman, Incompressible second-order fluids, Adv. in Appl. Mech. 8 (1964), 69-101.

[7] R. S. Rivlin and J. L. Ericksen, Stress-deformation relations for isotropic materials, J. Rational Mech. Anal. 4 (1955), 323-425.

[8] N. Rott, Unsteady viscous flow in the vicinity of a stagnation point, Quart. Appl. Math. 13 (1956), 444-451.

[9] A. C. Srivastava, Unsteady flow of a second-order fluid near a stagnation point, J. Fluid Mech. 24 (1966), no. 1, 33-39.

F. Labropulu: Department of Mathematics, Luther College, University of Regina, Regina, Saskatchewan, Canada S4S 0A2

E-mail address: fotini . 1abropu1u@uregina.ca

X. Xu: Department of Civil Engineering, University of Waterloo, Waterloo, Ontario, Canada N2L 3G1

E-mail address: xxu@engmai 1 . uwater $100 . \mathrm{ca}$

M. Chinichian: Faculty of Engineering, University of Regina, Regina, Saskatchewan, Canada S4S 0A2

E-mail address: mazdak_coop@yahoo.com 


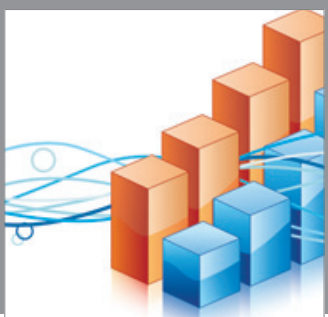

Advances in

Operations Research

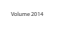

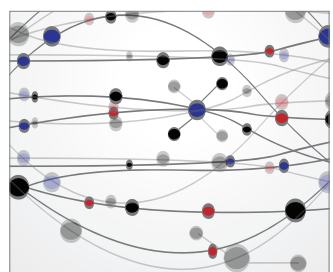

\section{The Scientific} World Journal
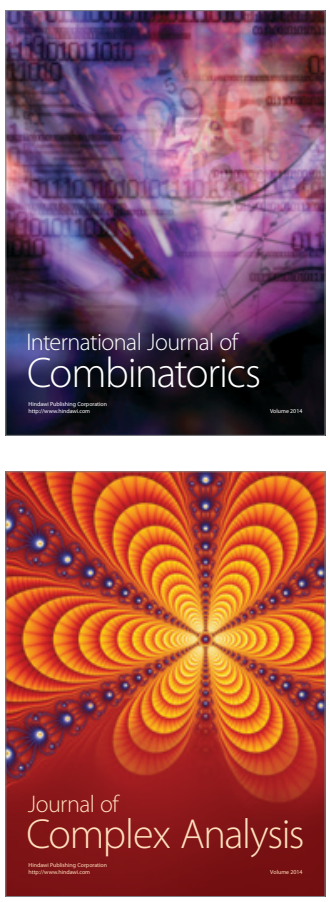

International Journal of

Mathematics and

Mathematical

Sciences
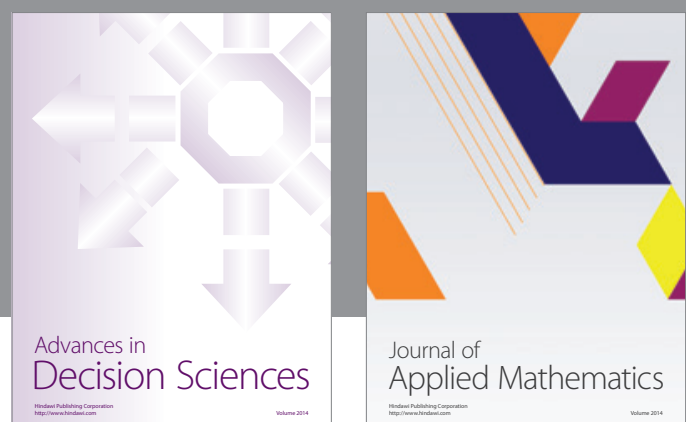

Journal of

Applied Mathematics
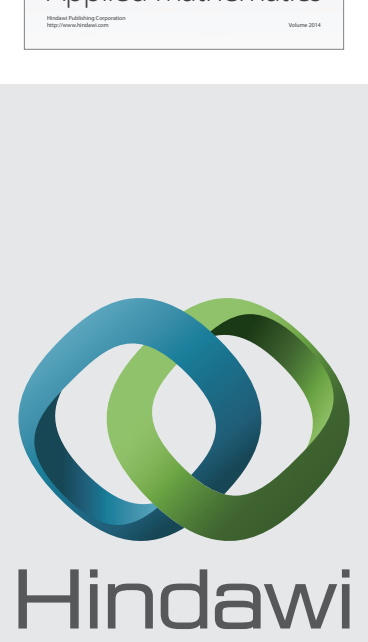

Submit your manuscripts at http://www.hindawi.com
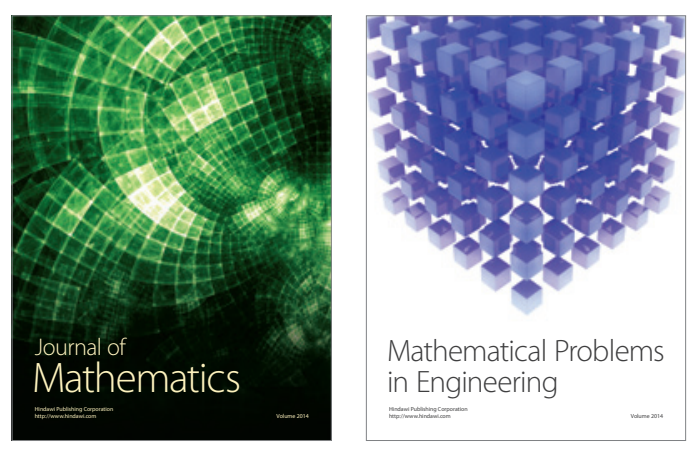

Mathematical Problems in Engineering
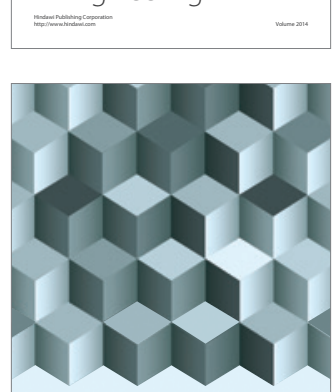

Journal of

Function Spaces
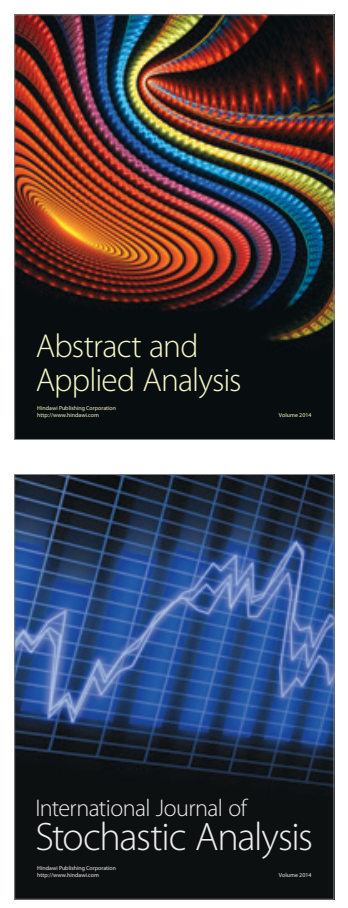

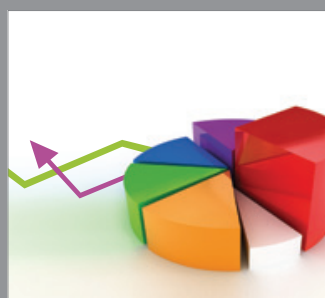

ournal of

Probability and Statistics

Promensencen
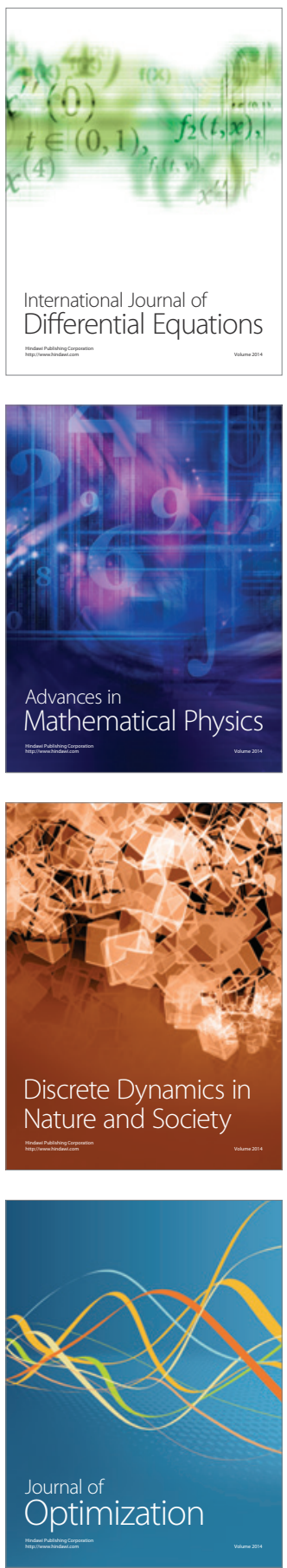